\title{
Isomorphisms of some reflexive algebras
}

\author{
by \\ JiAnkui Li (Shanghai) and Zhidong PAN (University Center, MI)
}

\begin{abstract}
Suppose $\mathcal{L}_{1}$ and $\mathcal{L}_{2}$ are subspace lattices on complex separable Banach spaces $X$ and $Y$, respectively. We prove that under certain lattice-theoretic conditions every isomorphism from $\operatorname{alg} \mathcal{L}_{1}$ to $\operatorname{alg} \mathcal{L}_{2}$ is quasi-spatial; in particular, if a subspace lattice $\mathcal{L}$ of a complex separable Banach space $X$ contains a sequence $E_{i}$ such that $\left(E_{i}\right)_{-} \neq X$, $E_{i} \subseteq E_{i+1}$, and $\bigvee_{i=1}^{\infty} E_{i}=X$ then every automorphism of $\operatorname{alg} \mathcal{L}$ is quasi-spatial.
\end{abstract}

1. Introduction. Let $X$ and $Y$ be separable complex Banach spaces and let $B(X, Y)$ be the set of all bounded linear maps from $X$ into $Y$. When $X=Y$, we use $B(X)$ instead of $B(X, Y)$. When $X$ is a Hilbert space, we use $H$ instead of $X$. For vector spaces $\mathcal{U}$ and $\mathcal{V}$, we write $L(\mathcal{U}, \mathcal{V})$ for the set of all linear maps from $\mathcal{U}$ to $\mathcal{V}$. By a subspace lattice on $X$, we mean a collection $\mathcal{L}$ of closed subspaces of $X$ with 0 and $X$ in $\mathcal{L}$ such that for every family $\left\{M_{r}\right\}$ of elements of $\mathcal{L}$, both $\bigcap M_{r}$ and $\bigvee M_{r}$ belong to $\mathcal{L}$. If the operations of meet and join distribute over each other for any collections of subspaces in $\mathcal{L}$, then $\mathcal{L}$ is said to be completely distributive. If $L \in \mathcal{L}$, we denote by $L_{-}$the subspace $\bigvee\{M \in \mathcal{L}: L \nsubseteq M\}$ and denote by $L_{+}$the subspace $\bigcap\{M \in \mathcal{L}: M \nsubseteq L\}$. For a subspace lattice $\mathcal{L}$ of $X$, we use alg $\mathcal{L}$ to denote the algebra of all operators on $X$ that leave members of $\mathcal{L}$ invariant.

For Hilbert spaces, a common practice is to disregard the distinction between a subspace and the orthogonal projection onto it. A Hilbert space subspace lattice $\mathcal{L}$ is called a commutative subspace lattice if it consists of mutually commuting projections. If $\mathcal{L}$ is a commutative subspace lattice then $\operatorname{alg} \mathcal{L}$ is called a $C S L$ algebra.

If $\mathcal{L}$ is a subspace lattice on $X$, we define $\mathcal{J}_{\mathcal{L}}=\{L \in \mathcal{L}: L \neq 0$ and $\left.L_{-} \neq X\right\}$. We say $\mathcal{J}_{\mathcal{L}}$ is sequentially dense in $X$ if there exists a sequence $E_{i} \in \mathcal{J}_{\mathcal{L}}$ such that $E_{i} \subseteq E_{i+1}$ and $\bigvee_{i=1}^{\infty} E_{i}=X$. Quasi-spatiality of isomorphisms has been studied in $[1,2,4,5]$. The main task of [4] is to show that if $\mathcal{L}$ is a commutative subspace lattice on a Hilbert space $H$ such that $\mathcal{J}_{\mathcal{L}}$ is

2000 Mathematics Subject Classification: Primary 47B47, 47L35.

Key words and phrases: subspace lattice, isomorphism, reflexive.

This work was completed with the support of NSF of China. 
sequentially dense in $H$ then every automorphism on $\operatorname{alg} \mathcal{L}$ is quasi-spatial. In this paper, we generalize the above result, with a relatively simpler proof, to non-commutative subspace lattices on Banach spaces; more specifically, we show that if $\mathcal{L}$ is any subspace lattice on a Banach space $X$ such that $\mathcal{J}_{\mathcal{L}}$ is sequentially dense in $X$ then every automorphism on $\operatorname{alg} \mathcal{L}$ is quasi-spatial. Our main result, Theorem 2.6, is stated in a slightly more general form; this also makes the presentation of the proof a little clearer.

2. The main result. For a subspace $E$ of a Banach space $X$, we define $E^{\perp}=\left\{f^{*} \in X^{*}:\left.f^{*}\right|_{E}=0\right\}$. For any $x \in X$ and $f^{*} \in X^{*}$, we use $x \otimes f^{*}$ to denote the rank-one operator satisfying $x \otimes f^{*}(u)=f^{*}(u) x$ for all $u \in X$. It follows from [3] that $x \otimes f^{*} \in \operatorname{alg} \mathcal{L}$ if and only if there exists an $L \in \mathcal{J}_{\mathcal{L}}$ such that $x \in L$ and $f^{*} \in\left(L_{-}\right)^{\perp}$. In the following, we suppose $\mathcal{L}_{1}$ and $\mathcal{L}_{2}$ are subspace lattices on Banach spaces $X$ and $Y$, respectively; and $\operatorname{alg} \mathcal{L}_{1}$ and $\operatorname{alg} \mathcal{L}_{2}$ are the corresponding subalgebras of $B(X)$ and $B(Y)$, respectively.

We will break the proof of the main result into a few lemmas.

Lemma 2.1. Suppose $\mathcal{J}_{\mathcal{L}_{2}}$ is sequentially dense in $Y, \psi$ is an isomorphism from $\operatorname{alg} \mathcal{L}_{2}$ to $\operatorname{alg} \mathcal{L}_{1}$, and $E \in \mathcal{J}_{\mathcal{L}_{1}}$. Then for any $x \in E$, there exist $K \in \mathcal{J}_{\mathcal{L}_{2}}, y \in K, h^{*} \in\left(K_{-}\right)^{\perp}$, and $0 \neq g^{*} \in X^{*}$ such that $\psi\left(y \otimes h^{*}\right)=x \otimes g^{*}$.

Proof. Take any $x \in E$ and $0 \neq l^{*} \in\left(E_{-}\right)^{\perp}$. Then $x \otimes l^{*} \in \operatorname{alg} \mathcal{L}_{1}$. Since $\psi$ is surjective, there exists a $B \in \operatorname{alg} \mathcal{L}_{2}$ such that $\psi(B)=x \otimes l^{*}$. Since $\mathcal{J}_{\mathcal{L}_{2}}$ is sequentially dense in $Y$, there exist a $K \in \mathcal{J}_{\mathcal{L}_{2}}$ and $w \in K$ such that $y=B w \neq 0$. Choose $0 \neq h^{*} \in\left(K_{-}\right)^{\perp}$ and set $A=\psi\left(w \otimes h^{*}\right)$ and $g^{*}=A^{*} l^{*}$. Then $\psi\left(y \otimes h^{*}\right)=\psi\left((B w) \otimes h^{*}\right)=\psi\left(B w \otimes h^{*}\right)=\psi(B) \psi\left(w \otimes l^{*}\right)=x \otimes l^{*} A=$ $x \otimes g^{*}$.

REMARK 2.2. Let $K$ be as in Lemma 2.1. From the proof of Lemma 2.1, one can see that, for any $L \in \mathcal{J}_{\mathcal{L}_{2}}$ with $K \subseteq L$, there exist $y_{1} \in L$, $h_{1}^{*} \in\left(L_{-}\right)^{\perp}$, and $0 \neq g_{1}^{*} \in X^{*}$ such that $\psi\left(y_{1} \otimes h_{1}^{*}\right)=x \otimes g_{1}^{*}$.

Lemma 2.3. Suppose $E_{i} \in \mathcal{J}_{\mathcal{L}_{1}}$ with $E_{i} \subseteq E_{i+1}, \bigvee_{i=1}^{\infty} E_{i}=X$, and $K_{i} \in \mathcal{J}_{\mathcal{L}_{2}}$ with $K_{i} \subseteq K_{i+1}$ and $\bigvee_{i=1}^{\infty} K_{i}=Y$. If $\phi$ is an isomorphism from $\operatorname{alg} \mathcal{L}_{1}$ to alg $\mathcal{L}_{2}$, then there exist $K_{n_{i}} \in \mathcal{J}_{\mathcal{L}_{2}}$ with $K_{n_{i}} \subseteq K_{n_{i+1}}, \bigvee_{i=1}^{\infty} K_{n_{i}}=$ $Y$, and injective $T_{i} \in L\left(E_{i}, Y\right)$ with $\operatorname{ran}\left(T_{i}\right) \subseteq K_{n_{i}}$ such that $\phi(A) T_{i} x=$ $T_{i} A x$ for every $x \in E_{i}$ and $A \in \operatorname{alg} \mathcal{L}_{1}$.

Proof. For any $0 \neq f_{i}^{*} \in\left(\left(E_{i}\right)_{-}\right)^{\perp}$, there exist $E_{m_{i}}$ and $x_{i} \in E_{m_{i}}$ such that $f_{i}^{*}\left(x_{i}\right)=1$. By Lemma 2.1 , there exist $y_{i} \in K_{n_{i}} \in \mathcal{J}_{\mathcal{L}_{2}}, h_{i}^{*} \in\left(\left(K_{n_{i}}\right)_{-}\right)^{\perp}$, and $0 \neq g_{i}^{*} \in X^{*}$ such that $\phi^{-1}\left(y_{i} \otimes h_{i}^{*}\right)=x_{i} \otimes g_{i}^{*}$. Since $E_{i} \subseteq E_{i+1}$ and $\bigvee_{i=1}^{\infty} E_{i}=X$, there exist $E_{p_{i}}$ and $u_{i} \in E_{p_{i}}$ such that $g_{i}^{*}\left(u_{i}\right)=1$. Define $T_{i} \in L\left(E_{i}, Y\right)$ by

$$
T_{i} x=\phi\left(x \otimes f_{i}^{*}\right) y_{i}, \quad \forall x \in E_{i}
$$


and define $S_{i} \in L\left(K_{n_{i}}, X\right)$ by

$$
S_{i} y=\phi^{-1}\left(y \otimes h_{i}^{*}\right) u_{i}, \quad \forall y \in K_{n_{i}} .
$$

It is clear from the definition of $T_{i}$ that $\operatorname{ran}\left(T_{i}\right) \subseteq K_{n_{i}}$.

For any $x \in E_{i}$,

$$
\begin{aligned}
S_{i} T_{i} x & =\phi^{-1}\left(T_{i} x \otimes h_{i}^{*}\right) u_{i}=\phi^{-1}\left(\left(\phi\left(x \otimes f_{i}^{*}\right) y_{i}\right) \otimes h_{i}^{*}\right) u_{i} \\
& =\left(x \otimes f_{i}^{*}\right) \phi^{-1}\left(y_{i} \otimes h_{i}^{*}\right) u_{i}=\left(x \otimes f_{i}^{*}\right)\left(x_{i} \otimes g_{i}^{*}\right) u_{i} \\
& =\left(x \otimes f_{i}^{*}\right) x_{i}=x .
\end{aligned}
$$

In particular, $T_{i}$ and $\left.S_{i}\right|_{V_{i}}$ are injective, where $V_{i}=\operatorname{ran}\left(T_{i}\right)$. Furthermore,

$$
\begin{aligned}
\phi(A) T_{i} x=\phi(A) \phi\left(x \otimes f_{i}^{*}\right) y_{i}=\phi\left(A x \otimes f_{i}^{*}\right) y_{i} & =T_{i} A x, \\
\forall x & \in E_{i}, A \in \operatorname{alg} \mathcal{L}_{1} .
\end{aligned}
$$

Similar to (2.1) and (2.2), we can construct $T_{i+1}$ and $S_{i+1}$; by Remark 2.2 we can assume $K_{n_{i}} \subseteq K_{n_{i+1}}$.

For any Banach space $X, f^{*} \in X^{*}$ and $E \subseteq X$, define

$$
\left[E \otimes f^{*}\right]_{X}=\left\{x \otimes f^{*}: x \in E\right\} \text {. }
$$

Lemma 2.4. Suppose $E_{i} \in \mathcal{J}_{\mathcal{L}_{1}}$ with $E_{i} \subseteq E_{i+1}, \bigvee_{i=1}^{\infty} E_{i}=X$, and $K_{i} \in \mathcal{J}_{\mathcal{L}_{2}}$ with $K_{i} \subseteq K_{i+1}, \bigvee_{i=1}^{\infty} K_{i}=Y$. If $\phi$ is an isomorphism from $\operatorname{alg} \mathcal{L}_{1}$ to alg $\mathcal{L}_{2}$, then for each $a_{i}^{*} \in\left(\left(E_{i}\right)_{-}\right)^{\perp}$, there is a $b_{i}^{*} \in Y^{*}$ such that $\phi\left(\left[E_{i} \otimes a_{i}^{*}\right]_{X}\right) \subseteq\left[Y \otimes b_{i}^{*}\right]_{Y}$.

Proof. Let $T_{i}$ be as in Lemma 2.3. Then by (2.4) we have

$$
\phi(A) T_{i} x=T_{i} A x, \quad \forall x \in E_{i}, A \in \operatorname{alg} \mathcal{L}_{1} .
$$

It follows that $B T_{i} x=T_{i} \phi^{-1}(B) x$ for $x \in E_{i}$ and $B \in \operatorname{alg} \mathcal{L}_{2}$. This implies that whenever $B$ is a rank-one operator, $\phi^{-1}(B)$ is also a rank-one operator, since $\bigvee_{i=1}^{\infty} E_{i}=X$ and $T_{i}$ is injective. By the symmetry of $X$ and $Y, \phi$ also maps rank-one operators to rank-one operators.

For each fixed $m$, fix $0 \neq x_{1} \in E_{m}$ and $0 \neq a_{m}^{*} \in\left(\left(E_{m}\right)_{-}\right)^{\perp}$ and suppose $\phi\left(x_{1} \otimes a_{m}^{*}\right)=y_{1} \otimes b_{m}^{*}$ for some $y_{1} \in Y$ and $b_{m}^{*} \in Y^{*}$. We will show

$$
\phi\left(\left[E_{m} \otimes a_{m}^{*}\right]_{X}\right) \subseteq\left[Y \otimes b_{m}^{*}\right]_{Y} .
$$

Take any $x_{2} \in E_{m}$ such that $\left\{x_{1}, x_{2}\right\}$ is linearly independent. Suppose $\phi\left(x_{2} \otimes a_{m}^{*}\right)=y_{2} \otimes c_{m}^{*}$ for some $y_{2} \in Y$ and $c_{m}^{*} \in Y^{*}$. We only need to show $\left\{b_{m}^{*}, c_{m}^{*}\right\}$ is linearly dependent.

Applying (2.5) with $A=x_{1} \otimes a_{m}^{*}$ and $A=x_{2} \otimes a_{m}^{*}$, respectively, we obtain

$$
b_{m}^{*}\left(T_{i} x\right) y_{1}=a_{m}^{*}(x) T_{i} x_{1}, \quad \forall x \in E_{i},
$$

and

$$
c_{m}^{*}\left(T_{i} x\right) y_{2}=a_{m}^{*}(x) T_{i} x_{2}, \quad \forall x \in E_{i},
$$


Since $E_{i} \subseteq E_{i+1}$ and $\bigvee_{i=1}^{\infty} E_{i}=X$, there exist $E_{i}$ and $x \in E_{i}$ such that $a_{m}^{*}(x) \neq 0$. Since $T_{i}$ is injective and $\left\{x_{1}, x_{2}\right\}$ is linearly independent, $\left\{T_{i} x_{1}, T_{i} x_{2}\right\}$ is linearly independent; so $\left\{y_{1}, y_{2}\right\}$ is linearly independent, by (2.6) and (2.7).

Since $\phi$ maps rank-one operators to rank-one operators, $\phi\left(\left(x_{1}+x_{2}\right) \otimes a_{m}^{*}\right)$ is a rank-one operator. Thus, $y_{1} \otimes b_{m}^{*}+y_{2} \otimes c_{m}^{*}=\phi\left(\left(x_{1}+x_{2}\right) \otimes a_{m}^{*}\right)$ is a rank-one operator. Since $\left\{y_{1}, y_{2}\right\}$ is linearly independent, $\left\{b_{m}^{*}, c_{m}^{*}\right\}$ is linearly dependent.

For a subspace $\mathcal{S}$ of $L(\mathcal{U}, \mathcal{V})$, define $\operatorname{ref}_{a}(\mathcal{S})=\{T \in L(\mathcal{U}, \mathcal{V})$ : $T x \in \mathcal{S} x, \forall x \in \mathcal{U}\}$. We say $\mathcal{S}$ is algebraically reflexive if $\operatorname{ref}_{\mathrm{a}}(\mathcal{S})=\mathcal{S}$. It is well known and not hard to show that every one-dimensional subspace of $L(\mathcal{U}, \mathcal{V})$ is algebraically reflexive.

Lemma 2.5. Assuming the same hypotheses and notations as in Lemma 2.3 , by rescaling $T_{i}$ we can have $\left.T_{i+1}\right|_{E_{i}}=T_{i}$ for $i=1,2, \ldots$.

Proof. Fix any $a_{i}^{*} \in\left(\left(E_{i}\right)_{-}\right)^{\perp}$ and $v \in Y$, and define $D \in L\left(E_{i}, Y\right)$ by $D x=\phi\left(x \otimes a_{i}^{*}\right) v$ for $x \in E_{i}$. If $D$ is not the zero operator then $D$ is injective; indeed, by Lemma 2.4, there exists $b_{i} \in Y^{*}$ such that $\phi\left(x \otimes a_{i}^{*}\right)=\lambda_{x} \otimes b_{i}^{*}$ for all $x \in E_{i}$. Since $\phi$ maps rank-one operators to rank-one operators, $\lambda_{x} \neq 0$ for all $0 \neq x \in E_{i}$. If $D$ is not the zero operator then $b_{i}^{*}(v) \neq 0$, so $D$ is injective; in particular, the operators $T_{i}$ defined by (2.1) are injective (which we already knew). By the symmetry of $X$ and $Y$, the operators $S_{i}$ defined by (2.2) are also injective.

Suppose $T_{i}, S_{i}, T_{i+1}$, and $S_{i+1}$ have been constructed as in Lemma 2.3. Then $S_{i+1} T_{i+1} x=x$ for all $x \in E_{i+1}$; in particular, $S_{i+1} T_{i+1} x=x$ for all $x \in E_{i}$. Let $V_{i}=\operatorname{ran}\left(T_{i}\right)$ and note that $V_{i} \subseteq K_{n_{i}} \subseteq K_{n_{i+1}}$. Consider $\left.S_{i}\right|_{V_{i}},\left.S_{i+1}\right|_{V_{i}} \in L\left(V_{i}, X\right)$. Since the one-dimensional subspace generated by the transformation $\left.S_{i}\right|_{V_{i}}$ is algebraically reflexive in $L\left(V_{i}, X\right)$ and

$$
\begin{aligned}
S_{i+1} T_{i} x & =\phi^{-1}\left(T_{i} x \otimes h_{i+1}^{*}\right) u_{i+1}=\phi^{-1}\left(\left(\phi\left(x \otimes f_{i}^{*}\right) y_{i}\right) \otimes h_{i+1}^{*}\right) u_{i+1} \\
& =\left(x \otimes f_{i}^{*}\right) \phi^{-1}\left(y_{i} \otimes h_{i+1}^{*}\right) u_{i+1}=\left(x \otimes f_{i}^{*}\right) t_{i+1} \\
& =f_{i}^{*}\left(t_{i+1}\right) x=f_{i}^{*}\left(t_{i+1}\right) S_{i} T_{i} x, \quad \forall x \in E_{i},
\end{aligned}
$$

where $t_{i+1}=\phi^{-1}\left(y_{i} \otimes h_{i+1}^{*}\right) u_{i+1}$, it follows that $\left.S_{i+1}\right|_{V_{i}}=\left.c_{i} S_{i}\right|_{V_{i}}$ for some scalar $c_{i}$. Since $S_{i+1}$ is injective, $c_{i} \neq 0$.

Replacing $S_{i+1}$ by $\left(1 / c_{i}\right) S_{i+1}$ and $T_{i+1}$ by $c_{i} T_{i+1}$ and still calling them $S_{i+1}$ and $T_{i+1}$, respectively, we have $\left.S_{i+1}\right|_{V_{i}}=\left.S_{i}\right|_{V_{i}}$, and for any $x \in E_{i}$, $S_{i+1} T_{i} x=S_{i} T_{i} x=x=S_{i+1} T_{i+1} x$. It follows that $T_{i+1} x=T_{i} x$ for all $x \in E_{i}$.

We say $\phi$ is quasi-spatial if there exists an injective linear transformation $T \in L(D(T), Y)$, where $D(T)$ is the domain of $T$ such that $D(T)$ is dense 
in $X$ and invariant under alg $\mathcal{L}_{1}$, the range of $T$ is dense in $Y$, and

$$
\phi(A) T x=T A x, \quad \forall x \in D(T), A \in \operatorname{alg} \mathcal{L}_{1} .
$$

Theorem 2.6. Suppose $\mathcal{J}_{\mathcal{L}_{1}}$ is sequentially dense in $X$ and $\mathcal{J}_{\mathcal{L}_{2}}$ is sequentially dense in $Y$. Then every isomorphism $\phi$ from $\operatorname{alg} \mathcal{L}_{1}$ to $\operatorname{alg} \mathcal{L}_{2}$ is quasi-spatial; in particular, $\phi$ preserves ranks of operators.

Proof. By the assumptions, there exist $E_{i} \in \mathcal{J}_{\mathcal{L}_{1}}$ with $E_{i} \subseteq E_{i+1}$, $\bigvee_{i=1}^{\infty} E_{i}=X$, and $K_{i} \in \mathcal{J}_{\mathcal{L}_{2}}$ with $K_{i} \subseteq K_{i+1}, \bigvee_{i=1}^{\infty} K_{i}=Y$. Now we can construct $T_{i}$ as in Lemma 2.3, with modifications as in Lemma 2.5. Let $E=\bigcup_{i=1}^{\infty} E_{i}$, the non-closed union of $E_{i}$, so $E$ is dense in $X$. Clearly, $E$ is invariant under $\operatorname{alg} \mathcal{L}_{1}$, and if $x \in E$ then $x \in E_{i}$ for some $i$. Define $T x=T_{i} x$. By the agreement among $T_{i}$, it follows that $T$ is a well-defined, injective, linear transformation on $E$; moreover, $\phi(A) T x=T A x$ for all $x \in E$ and $A \in \operatorname{alg} \mathcal{L}_{1}$. Let $\operatorname{ran}(T)$ be the range of $T$ and $K=\bigcup_{i=1}^{\infty} K_{i}$. Clearly $K$ is dense in $Y$ and $\operatorname{ran}(T) \subseteq K$; we will show $\operatorname{ran}(T)=K$. Take any $y \in K$. There exists $K_{n_{i}}$ such that $y \in K_{n_{i}}$. By (2.2) of Lemma 2.3, $S_{i} y=\phi^{-1}\left(y \otimes h_{i}^{*}\right) u_{i} \in E_{p_{i}} \in E$. By (2.1) of Lemma 2.3,

$$
\begin{aligned}
T S_{i} y & =T_{p_{i}} S_{i} y=\phi\left(\phi^{-1}\left(y \otimes h_{i}^{*}\right) u_{i} \otimes f_{p_{i}}^{*}\right) y_{p_{i}}=\left(y \otimes h_{i}^{*}\right) \phi\left(u_{i} \otimes f_{p_{i}}^{*}\right) y_{p_{i}} \\
& =h_{i}^{*}\left(\phi\left(u_{i} \otimes f_{p_{i}}^{*}\right) y_{p_{i}}\right) y=\mu_{i} y,
\end{aligned}
$$

where $\mu_{i}=h_{i}^{*}\left(\phi\left(u_{i} \otimes f_{p_{i}}^{*}\right) y_{p_{i}}\right)$. Since $T_{p_{i}}$ and $S_{i}$ are injective, $\mu_{i} \neq 0$. Now $T\left(\mu_{i}^{-1} S_{i} y\right)=y$, so $\operatorname{ran}(T)=K$.

Rank-preserving follows from (2.8) directly.

The following corollary is the main result of [4]. A special case of the corollary was proved earlier in [1] with an additional hypothesis of subspace lattices being completely distributive.

Corollary 2.7 ([4, Theorem 17]). Suppose $\mathcal{L}_{1}$ and $\mathcal{L}_{2}$ are commutative subspace lattices on a Hilbert space $H$ and $\mathcal{J}_{\mathcal{L}_{1}}$ is sequentially dense in $H$. Then every isomorphism from $\operatorname{alg} \mathcal{L}_{1}$ to $\operatorname{alg} \mathcal{L}_{2}$ is quasi-spatial.

Proof. By $[4$, Theorem C $]$, we can assume $\mathcal{L}_{1}=\mathcal{L}_{2}$. Now the conclusion follows from Theorem 2.6.

Remark: The hypotheses in [4, Theorem 17] are stated differently from Corollary 2.7, but it is easy to check that they are equivalent.

TheOREM 2.8. If $\mathcal{L}_{1}$ is a subspace lattice with $X_{-} \neq X$ and $\mathcal{L}_{2}$ is a subspace lattice with $Y_{-} \neq Y$, then every isomorphism from alg $\mathcal{L}_{1}$ to $\operatorname{alg} \mathcal{L}_{2}$ is spatially implemented and every bounded isomorphism from $\operatorname{alg} \mathcal{L}_{1}$ to $\operatorname{alg} \mathcal{L}_{2}$ is spatially implemented by a bounded operator.

Proof. Suppose $\phi$ is an isomorphism from alg $\mathcal{L}_{1}$ to alg $\mathcal{L}_{2}$. Take $E_{i}=X$ and $K_{i}=Y$, then the hypotheses of Theorem 2.6 are satisfied. Let $T_{i}$ be defined by (2.1) and $S_{i}$ be defined by (2.2) in Lemma 2.3. By (2.3), 
$S_{i} \in L(Y, X)$ is surjective. By the first paragraph of the proof of Lemma 2.5, $S_{i}$ is injective, so $S_{i}$ has an inverse. Now the equality $S_{i} T_{i} x=x$ for all $x \in E_{i}(=X)$ implies $T_{i}$ is invertible with $T_{i}^{-1}=S_{i}$. Finally, (2.5) of Lemma 2.4 implies $\phi$ is spatially implemented. If $\phi$ is bounded, then so are $T_{i}$ and $S_{i}$.

COROLlary 2.9. If $\mathcal{L}$ is a subspace lattice on a Hilbert space $H$ with $0_{+} \neq 0$, then every automorphism of $\operatorname{alg} \mathcal{L}$ is spatial.

Proof. Suppose $\mathcal{L}$ satisfies $0_{+} \neq 0$ and $\phi$ is an automorphism of alg $\mathcal{L}$. Let $\mathcal{L}^{\perp}=\{I-L: L \in \mathcal{L}\}$, where $I$ is the identity operator on $H$. Then $\mathcal{L}^{\perp}$ satisfies $H_{-} \neq H$.

Define $\phi^{*}\left(A^{*}\right)=(\phi(A))^{*}$ for $A^{*} \in \operatorname{alg} \mathcal{L}^{\perp}$. Then $\phi^{*}$ is an automorphism of alg $\mathcal{L}^{\perp}$. By Theorem 2.8, we have $\phi^{*}\left(A^{*}\right)=(\phi(A))^{*}=T A^{*} T^{-1}$ for some $T \in B(H)$. So $\phi$ is spatial.

\section{References}

[1] F. Gilfeather and R. L. Moore, Isomorphisms of certain CSL algebras, J. Funct. Anal. 67 (1986), 264-291.

[2] J. Li and O. Panaia, Algebraic isomorphisms and $\mathcal{J}$-subspace lattices, Proc. Amer. Math. Soc. 133 (2005), 2577-2587.

[3] W. E. Longstaff, Strongly reflexive lattices, J. London Math. Soc. 11 (1975), 491-498.

[4] R. L. Moore, Isomorphisms for CSL algebras, Indiana Univ. Math. J. 52 (2003), 687-702.

[5] O. Panaia, Algebraic isomorphisms and finite distributive subspace lattices, J. London Math. Soc. 59 (1999), 1033-1048.

Department of Mathematics

East China University of Science and Technology

Shanghai 200237, P.R. China

E-mail: jiankuili@yahoo.com
Department of Mathematics

Saginaw Valley State University University Center, MI 48710, U.S.A.

E-mail: pan@svsu.edu

Received February 22, 2007

Revised version February 25, 2008 